\title{
Catalyst layer doped with phosphotungstic acid for degradation mitigation in Polymer Electrolyte Membrane Fuel Cells
}

Guang-Ying Chen ${ }^{a}$, Cheng Wang ${ }^{a}{ }^{*}$, Yi-Jie Lei $^{a}{ }$, Jianbo Zhang $^{b}$, Zong-Qiang Mao $^{a}$, Jian-Wei Guo $^{a}$ Jian-Long Wang ${ }^{a}$

${ }^{a}$ Institute of Nuclear and New Energy Technology, Tsinghua University, Beijing 100084, China

${ }^{\mathrm{b}}$ State Key Laboratory of Automotive Safety and Energy, Tsinghua University, Beijing 100084, China

Corresponding author: Cheng Wang, ${ }^{a}$ *

Tel: +8601062797595, Fax: +8601062797595

E-mail address: wangcheng@tsinghua.edu.cn

Postal address: Institute of Nuclear and New Energy Technology, Tsinghua University, Beijing

100084, China

\section{Abstract:}

The aim of this study was to enhance durability and performance of Polymer Electrolyte Membrane Fuel Cells (PEMFCs) for transportation application. A modified catalyst coated membrane (CCM) with phosphotungstic acid (PTA) in catalyst layer was fabricated using a heating ultrasonicspray method, and durability study of both the modified CCM and traditional CCM was carried out according to the DOE CCM/stack durability protocol. During the accelerated stress test of CCM durability, cyclic voltammetry, polarization curves, linear sweep voltammetry and electrochemical impedance spectroscopy were employed for diagnosis of the CCM performances. The experimental results indicate that the power density of the modified CCM decreased only $14 \%$ while the traditional CCM decreased 33\% after 100-hour aging time, which confirms that addition of PTA in the catalyst layer of CCM is an effective way to improve the durability for CCM attributed to the enhancement of intermediates removal and the increasing of proton transfer channels by using the PTA excellent properties, both redox property and proton conductivity.

Keywords: Proton exchange membrane fuel cell; Durability; Phosphotungstic acid; Accelerated stress test; Mitigation 


\section{Introduction}

PEMFCs are considered to be a promising energy conversion device for transportation and stationary applications due to their high efficiency, high energy density and zero or low pollution [1]. Durability, apart from performance and cost requirements is one of the significant barriers to PEMFC industrialization. In the past few years, numerous studies have shown that several factors including proton exchange membrane thinning, catalyst layer degradation and gas diffusion layer degradation can affect the durability of a PEMFC. More and more experimental results have shown catalyst layer degradation in the component of CCM is one of the most critical factors for performance degradation in PEMFC [2]. The catalyst layer in CCM for current PEMFC is mainly composed of platinum based catalyst and Nafion ionomer used generally in the membrane. Without the Nafion ionomer addition, the majority of the platinum based catalyst sites are inactive [3]. It is known that the intermediates such as hydrogen peroxide, $\mathrm{OH}$ radicals produced during the fuel cell working process are potential species that damage perfluorosulfonic acid polymer in Nafion ionomer by attacking at the alpha hydrogen [4]. The dissolution and chemical degradation of Nafion ionomer in the catalyst layer might be more severe than that in the membrane because of it is located adjacent to the fuel cell reaction active sites. Xie et al. [5] revealed the degradation of the ionomer network in the catalyst layer after 500h of operation under high humidity and constant current using cell impedance trends. Zhang et al. [6] detected the catalyst layer ionomer degradation after $300 \mathrm{~h}$ of fuel cell operation using XPS quantitative analysis. According to their report, the catalyst layer surface concentration of fluorine reduced from $50.1 \%$ to $38.9 \%$, consistent with a decrease of $\mathrm{CF}_{3}$ and $\mathrm{CF}_{2}$ species and an increase in oxidized forms of carbon. Which undoubtedly was a proof for the Nafion ionomer change in catalyst layer. Therefore, increasing catalyst layer durability is becoming a major challenge and growing focus of research attention in 
PEMFC durability studies. Possible mitigation methods for degradation of Nafion ionomer in the catalyst layer include reducing peroxide from electrochemical reaction by using radical inhibitors or peroxide-decomposition catalysts deposited within catalyst layer to decrease the severity of radical attacks. Research results show that solid heteropoly acids have excellent redox property and proton conductivity, and the addition of heteropoly acid to Nafion membrane is a significant route to achieve the objective of improving durability attributed to the enhancement of intermediates removal and increase of proton transfer channels [7]. According to this principle of the degradation mitigation for Nafion membrane, we expect that adding heteropoly acid into the catalyst layer is also an effective way to improve the durability for CCM. Zhou et al. [8] developed a novel proton exchange membrane using phosphotungstic acid ( $\mathrm{PTA}, \mathrm{H}_{3} \mathrm{PW}_{12} \mathrm{O}_{40} \cdot \mathrm{xH}_{2} \mathrm{O}$ ) as proton carrier and mesoporous silica as matrix (PTA-meso-silica). The study provided us the idea of using phosphotungstic acid as both proton carrier and peroxide-decomposition catalyst to improve the durability of PEMFC without apparent decrease in proton conductivity.

In this study, the PEMFC durability was investigated by impregnating phosphotungstic acid into catalyst layer in CCM (the modified CCM). The DOE PEMFC durability test protocol [2] was employed to evaluate the durability difference between the modified CCM and the traditional CCM (no phospho-tungstic acid). During durability test of CCMs, cyclic voltammetry (CV), polarization curves (IV), linear sweep voltammetry (LSV) and electrochemical impedance spectroscopy (EIS) were adopted for performance status diagnosis of PEMFC.

\section{Experimental}

\subsection{Preparation of CCM}

The CCMs were fabricated using a heating ultrasonic-spray method, where the catalyst ink was 
sprayed directly onto the Nafion XL membrane (DuPont, USA) at a temperature of $100{ }^{\circ} \mathrm{C}$. The catalyst ink was prepared by mixing Pt/C catalyst (Johnson Matthey, Pt $40 \mathrm{wt} \%$ ), isopropanol, and a Nafion ionomer solution (DuPont, 5wt\%), with or without 3wt\% phosphotungstic acid (PTA, $\mathrm{H}_{3} \mathrm{PW}_{12} \mathrm{O}_{40} \cdot \mathrm{xH}_{2} \mathrm{O}$, reagent grade obtained from Sigma Aldrich, FW2880.05) adding respectively. The two categories of CCMs (traditional CCM and modified CCM) were prepared with the following membrane and electrode components given in Table1.

Table 1. Specifications of CCM

\subsection{Accelerated stress test}

The prepared CCMs with active area of $25 \mathrm{~cm}^{2}$ were sandwiched between two gas diffusion layers (SGL-25BC, Germany), then assembled into a single fuel cell using graphite bipolar plates with serpentine flow channels (Shanghai Hong Feng Industrial Company Limited), and the clamping force of the single cell was $5 \mathrm{~N} \cdot \mathrm{m}$ by eight lubricated bolts. This single fuel cell was connected to the HS150 Fuel Cell Test Equipment (Hephas Energy Co., Ltd). For the CCM accelerating degradation test, the fuel cell was evaluated at $80{ }^{\circ} \mathrm{C}$ with pure Air and $\mathrm{H}_{2}(99.999 \%)$ being used as cathode and anode reactant respectively. The current density and gas flow rate were changed every 30 seconds, and a test cycle finished after 20 test points. The complete operational protocol is presented schematically in Table 2 and diagrams of the cycles for accelerated stress test are shown in figure 1.

Table 2. DOE CCM/stack durability protocol
a) reactant gas flow rate
b) current density

Fig. 1 Cycle curves for accelerated stress test

\subsection{Electrochemical characterization}


At $0,100,200$, and $300 \mathrm{~h}$, the accelerating stress test was paused to conduct characterizations of CCM degradations, such as CV, IV, LSV and EIS. And the electrochemical performances were measured by an electrochemical workstation (ZAHNER IM6ex). During the CV and LSV measurements humidified hydrogen $\left(300 \mathrm{~mL} / \mathrm{min}\right.$, dew point: $\left.68{ }^{\circ} \mathrm{C}\right)$ and $\mathrm{N}_{2}(500 \mathrm{~mL} / \mathrm{min}$, dew point: $68{ }^{\circ} \mathrm{C}$ ) were supplied to the anode and the cathode respectively. The scan range is $0.05-1.4 \mathrm{~V}$ for $\mathrm{CV}$ with scan rate of $20 \mathrm{~m} \mathrm{~V} / \mathrm{s}$, while the scan range is $0.05-0.6 \mathrm{~V}$ with $1 \mathrm{~m} \mathrm{~V} / \mathrm{s}$ rate for LSV. The EIS was conducted at a constant current of $200 \mathrm{~mA} / \mathrm{cm}^{2}$ with an AC frequency range of $100 \mathrm{kHz}-0.05 \mathrm{~Hz}$ and an amplitude of $5 \mathrm{mV}$.

\section{Results and discussion}

\subsection{Overall performance degradation in accelerated stress test}

Fig. 2 shows the overall performance change of the traditional and modified CCM during the aging time from 0 to $6000 \mathrm{~min}$. From the top to the bottom the three parts of the figure exhibit the voltage behaviors of the two CCMs under different constant current operation of $0.02 \mathrm{~A} / \mathrm{cm}^{2}, 0.1 \mathrm{~A} / \mathrm{cm}^{2}$ and $1.2 \mathrm{~A} / \mathrm{cm}^{2}$ respectively. It can be seen that the voltage distinction between the modified and traditional CCM became more and more obvious with the increase of current density. Furthermore, during the aging time from 0 to $6000 \mathrm{~min}$, the performance of modified CCM was better than the traditional $\mathrm{CCM}$ under the constant current operation of $0.02 \mathrm{~A} / \mathrm{cm}^{2}, 0.1 \mathrm{~A} / \mathrm{cm}^{2}$ and $1.2 \mathrm{~A} / \mathrm{cm}^{2}$, respectively. Even though the performance of the two CCM was almost the same under lower constant current operation of $0.02 \mathrm{~A} / \mathrm{cm}^{2}$ during the aging time from 0 to $1000 \mathrm{~min}$. From the result, an obvious conclusion can be summarized that the modified CCM showed an enhanced performance and stability over the traditional one, especially at higher current density. And the reasons for the performance degradation of the traditional CCM might be the main and the side chains of perfluorosulfonic acid 
ionomer attacked by free radical [9]. The free radicals were generated from the hydrogen peroxide byproduct during long operation process [9]. However, the addition of PTA to catalyst layer in CCM is an effective method to improve durability and performance. Three hydrogens in PTA molecular structure (as shown in Fig. 3) as hydrogen bonds forming of $\mathrm{O}-\mathrm{H} . . \quad$ O are connected with terminal oxygen in Keggin structure, and have excellent ability of scavenging free hydroxyl radicals. In addition, PTA effectively bridges the hydrophilic clusters and decreases the activation energy for proton hopping. Therefore, the modified CCM showed an enhanced stability and performance attributed to the enhancement of intermediates removal and increase of proton transfer channel.

Fig. 2 Overall performance of traditional CCM and modified CCM during accelerated stress test

Fig. 3 Molecular structure of the phospho-tungstic acid

\subsection{Performance comparison of polarization curves}

Fig.4 plots the potential (voltage) as a function of the current density of the modified CCM and traditional CCM at different aging time. Initially, the performance of modified and traditional CCM were almost the same at low current density (activation zone) while the modified CCM exhibiting better performance at higher current density. The power density of the traditional CCM decreased from $0.43 \mathrm{~W} / \mathrm{cm}^{2}$ to $0.29 \mathrm{~W} / \mathrm{cm}^{2}$ (@0.6 V) with 33\% decay after 100-hour aging time, while the power density of the modified CCM only decreased from $0.5 \mathrm{~W} / \mathrm{cm}^{2}$ to $0.43 \mathrm{~W} / \mathrm{cm}^{2}$, and $0.4 \mathrm{~W} / \mathrm{cm}^{2}$ (@0.6V) with $14 \%$ or $20 \%$ decay after 100 -hour or 300 -hour aging time, respectively. These results clearly indicated that the PTA modified CCM could improve the durability and performance of the CCM, for the reason that the addition of PTA could mitigate the degradation of ionomer in catalyst layer by peroxide attack and enhance proton conductivity. 
Fig.4 Polarization curves of traditional CCM and the modified CCM

(70 ${ }^{\circ} \mathrm{C}$ operation temperature and $80 \% \mathrm{RH}$ with the stoichiometry of air and $\mathrm{H}_{2}$ at 2.5 and 1.5 respectively)

\subsection{Analysis of cyclic voltammetry}

The CV curves of modified CCM and traditional CCM at different time are shown in Fig.5, from which proton adsorption/desorption on the platinum surface can be monitored at potentials lower than $0.4 \mathrm{~V}$. And the EASA (electrochemically active surface area) of the platinum catalysts can subsequently be calculated using the area under the hydrogen adsorption / desorption peaks as shown

in Table 3. Formula used is given as: $\mathrm{ECA}=($ Area under the peak $) /[$ scan rate in $\mathrm{V} / \mathrm{s}) *\left(210 \mathrm{C} / \mathrm{cm}^{2}-\right.$ Pt)*Total Pt loading in $\mathrm{mg}$ )]. It's obvious that the hydrogen desorption peak areas of traditional and modified CCM both decreased gradually during the degradation test, and after 100-hour aging time the EASA of modified CCM didn't show higher degradation rate than the traditional one, but it led to decreasing in EASA with the addition of PTA in the catalyst layer. Decrease of EASA might be due to the fact that PTA in the catalyst layer can get adsorbed on the catalyst sites, rendering the sites inactive for redox reaction.

Fig. 5-Cyclic voltammetry curves of the traditional CCM and the modified CCM

Table 3. EASAs of CCMs

\subsection{Analysis of linear sweep voltammetry}

Fig. 6 shows the $\mathrm{H}_{2}$ permeability of the traditional and the modified CCM after different aging time. It can be observed that the hydrogen crossover current density of traditional CCM and modified CCM increased with the aging time. The hydrogen crossover current density of modified CCM 
increased to $2.4 \mathrm{~mA} / \mathrm{cm}^{2}$ after 300-hour aging time, which closed to the normal level of $2.5 \mathrm{~mA} / \mathrm{cm}^{2}$. However, the traditional CCM increased to $2.75 \mathrm{~mA} / \mathrm{cm}^{2}$ after only 100 -hour aging time which indicated that the proton exchange membrane in the traditional CCM started degradation. The result can be attributed to the former experimental evidence that the main chain or side chain of the membrane were attacked by radicals which caused the membrane degradation and hydrogen permeation. On the contrary, as to the modified CCM, the peroxide was decomposed and the radical generation was restrained by the addition of PTA in catalyst layer, therefore the proton exchange membrane degradation was mitigated.

Fig. 6-Linear sweep voltammetry curves of the traditional CCM and the modified CCM

\subsection{Analysis of the AC impedance}

EIS is a powerful technique to characterize the resistance of PEMFC. From the analysis of the EIS curves shown in Fig. 7, it is found that the ohmic resistance was higher for the modified CCM than the traditional one from the very beginning, due to the addition of PTA in catalyst layer, but the ohmic resistance of the both CCMs didn't show significant change with the aging time. The diameter of the kinetic loop, which corresponded to the charge transfer resistance of the fuel cell consisting of the traditional or modified CCM, increased with the aging time as shown in Table. 4. The charge transfer resistance of the modified CCM was smaller than the traditional one at $0 \mathrm{~h}$, which even at 300 $\mathrm{h}$ was smaller than that of the traditional one at $100 \mathrm{~h}$. The impedance results show that the modified CCM owned better charge transfer resistance compared to the traditional one according to the decreased semicircle width in the Nyquist plots. Besides, it also exhibits less decreasing rate than the traditional one with the aging time, which indicates that the electrochemical activity of catalyst layer can be enhanced by the addition of PTA, the charge transfer resistance can be reduced, and the 
membrane proton conductivity degradation can also be mitigated.

Fig. 7 Nyquist plots of the traditional CCM and the modified CCM

Table 4. The charge transfer resistance of CCMs

\section{Conclusions}

In this study, a simple and effective approach is developed to improve the durability and properties of PEMFC by impregnating PTA into the catalyst layer in CCM through heating ultrasonicspray method. The results show that the modified CCM with the addition of PTA exhibited better performance and durability than the traditional $\mathrm{CCM}$ over the accelerating degradation (especially at higher current density) and polarization test, in which the power density of the modified CCM decreases only $14 \%$ while the traditional CCM decreases 33\% after 100-hour aging time. Although the addition of PTA decreases the EASA compared to the traditional one, it also decreases the degradation rate over the aging time test. The hydrogen crossover current density of the modified CCM is also lower than the traditional one throughout the accelerating degradation. Besides, adding PTA to the catalyst layer of the CCM can also lower the charge transfer resistance with negligible rising of ohmic resistance. The improvement owes to the addition of PTA which could decompose the peroxide, thus the radical attack to proton exchange membrane polymer chain and end groups (such as sulfonic group) can be mitigated. Apart from used as proton carrier in proton exchange membrane, PTA can also be impregnated into the catalyst layer in CCM as peroxide-decomposition catalyst to improve the durability and performance of PEMFC.

\section{Acknowledgement:}


This work is financially supported by the National Science Foundation of China (NSFC) (Grant No. U1462112, 21573122), the National Key Basic Research Program of China (Grant No. 2012CB215401) and the Program of International ST Cooperation (Grant No. 2013DFG41460, 2013DFG60080).

\section{References:}

[1] Wang Cheng, Wang Shubo, Zhang Jianbo, et al. The Durability Research on the Proton Exchange Membrane Fuel Cell for Automobile Application, PROGRESS IN CHEMISTRY, 2015, 27(4): 424435.

[2] Liu Mingyang, Wang Cheng, Xie Fucheng, et al. A polymer electrolyte fuel cell life test using accelerating degradation technique, INTERNATIONAL JOURNAL OF HYDROGEN ENERGY,2013,38(25):11011-11016.

[3] Song Ying, Xu Hui, Wei Yu, et al. Dependence of high-temperature PEM fuel cell performance on Nafion content, Journal of Power Sources, 2006, 154:138-144.

[4] Cheng Chen, Thomas F. Fuller. The effect of humidity on the degradation of Nafion membrane, Polymer Degradation and Stability, 2009, 94:1436-1447.

[5] J. Xie, D.L.Wood III, K.L. More, et al. Microstructural changes of membrane electrode assemblies during PEFC durability testing at high humidity conditions, J. Electrochem. Soc., 2005, 152: A1011A1020.

[6] Zhang Feng-Yuan, Advani Suresh G, Prasad Ajay K, et al. Quantitative characterization of catalyst layer degradation in PEM fuel cells by X-ray photoelectron spectroscopy, ELECTROCHIMICA ACTA, 2009, 54(16):4025-4030.

[7] Darling RM, Meyers JP. Kinetic model of platinum dissolution in PEMFCs, JOURNAL OF THE 
ELECTROCHEMICAL SOCIETY, 2003, 150(11): A1523-A1527.

[8] Zhou Yuhua, Yang Jing, Su Haibin, Zeng Jie, Jiang Sanping, Goddard William A. Insight into proton transfer in phosphotungstic acid functionalized mesoporous silica-based proton exchange membrane fuel cell, Journal of The American Chemical Society, 2014, 136: 4954-4964.

[9] P. Choi, N. H. Jalani, T. M. Thampan, and R. Datta. Consideration of thermodynamic, transport, and mechanical properties in the design of polymer electrolyte membranes for higher temperature fuel cell operation, J. Polym. Sci., Part B: Polym. Lett. , 2006, 44:2183 -2200. 

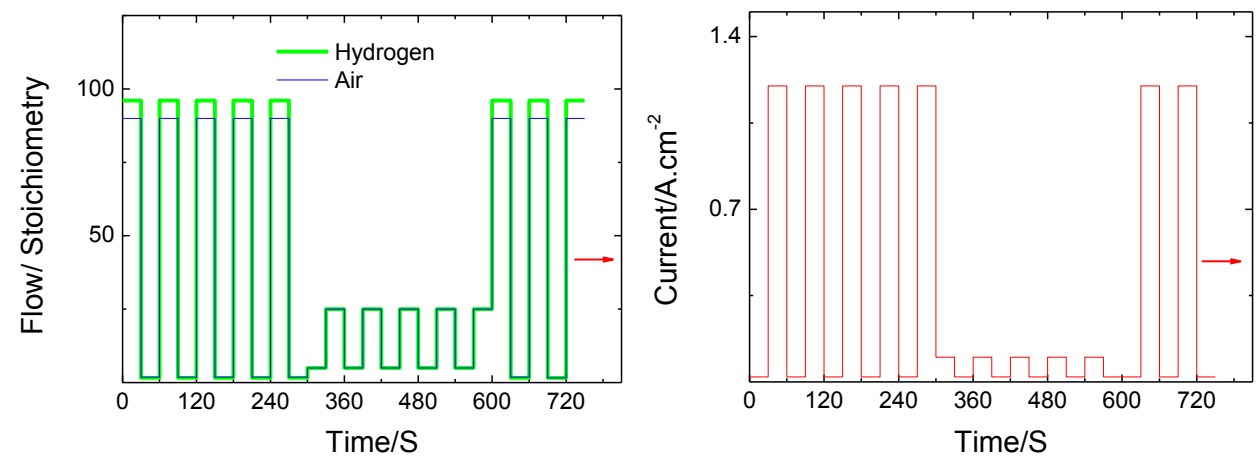

Fig. 1

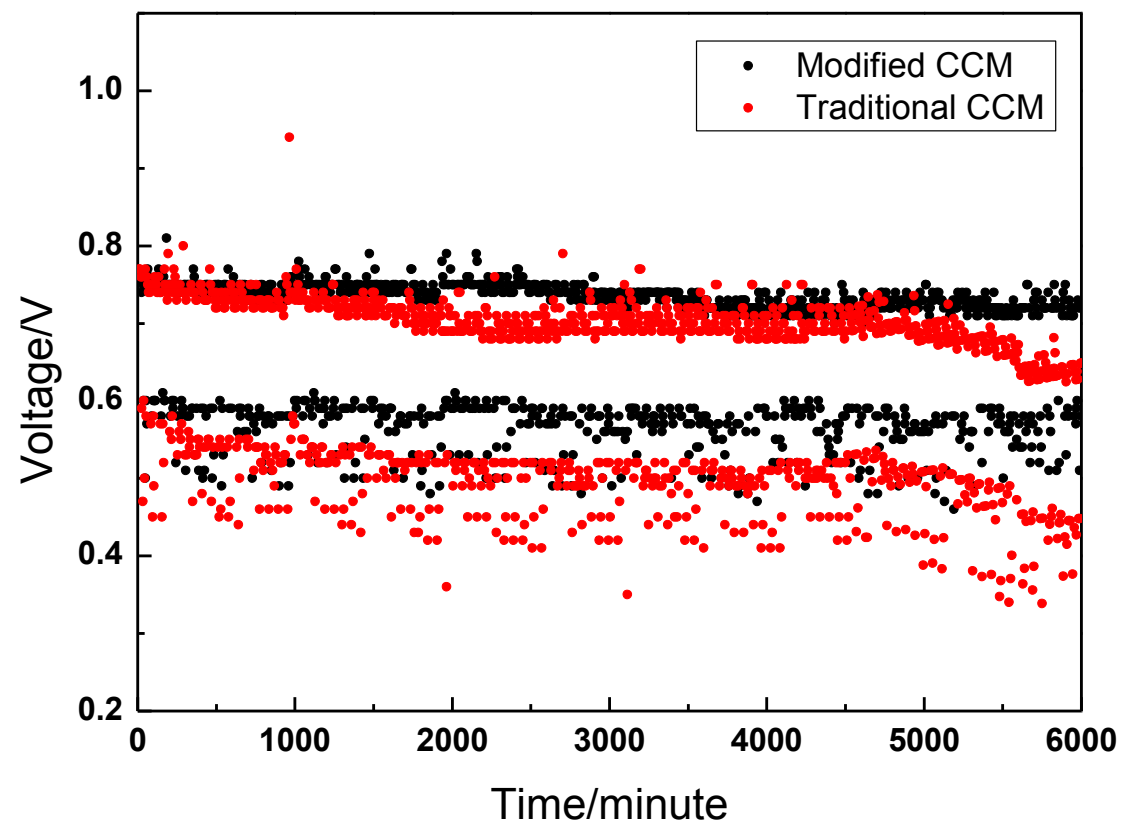

Fig. 2

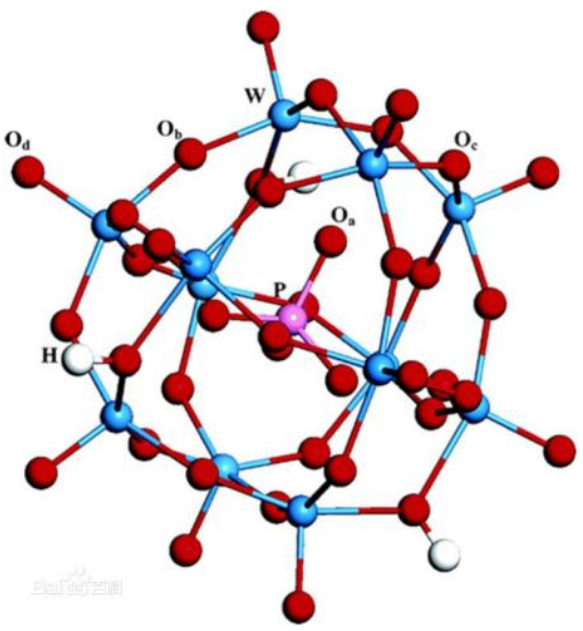

Fig. 3 


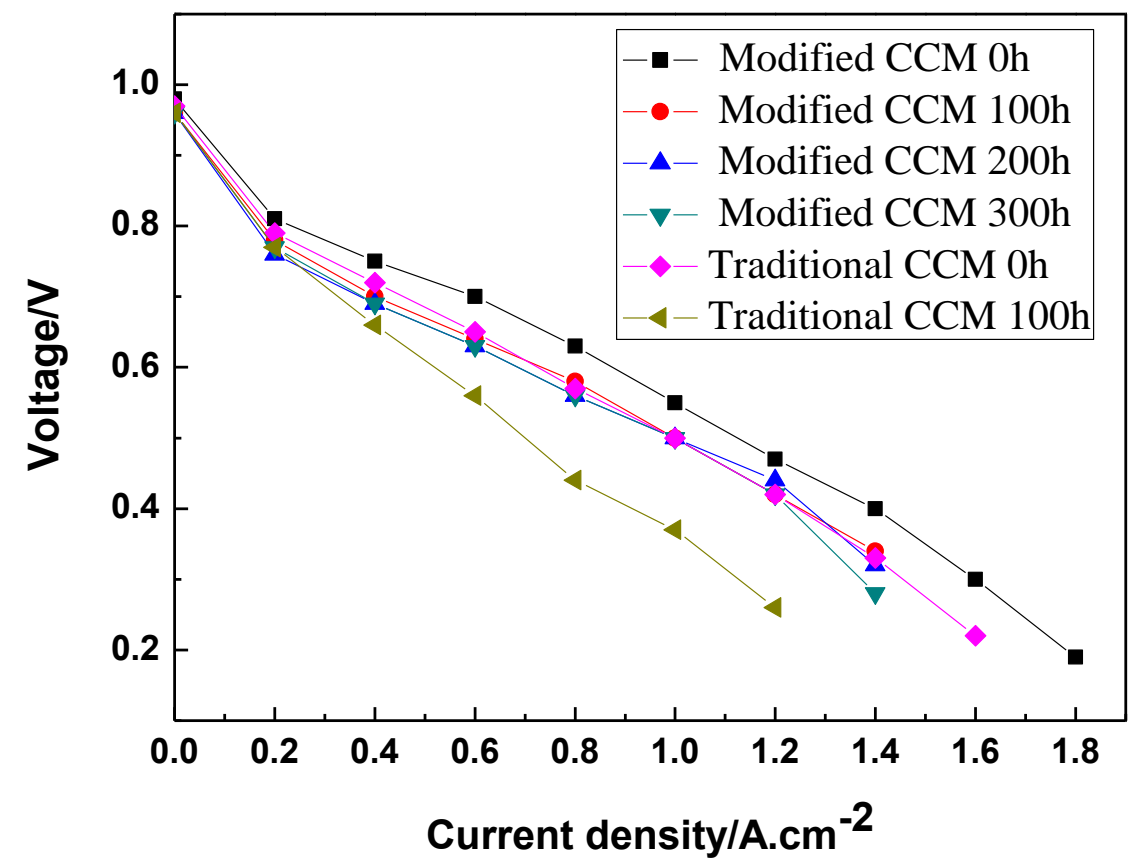

Fig. 4

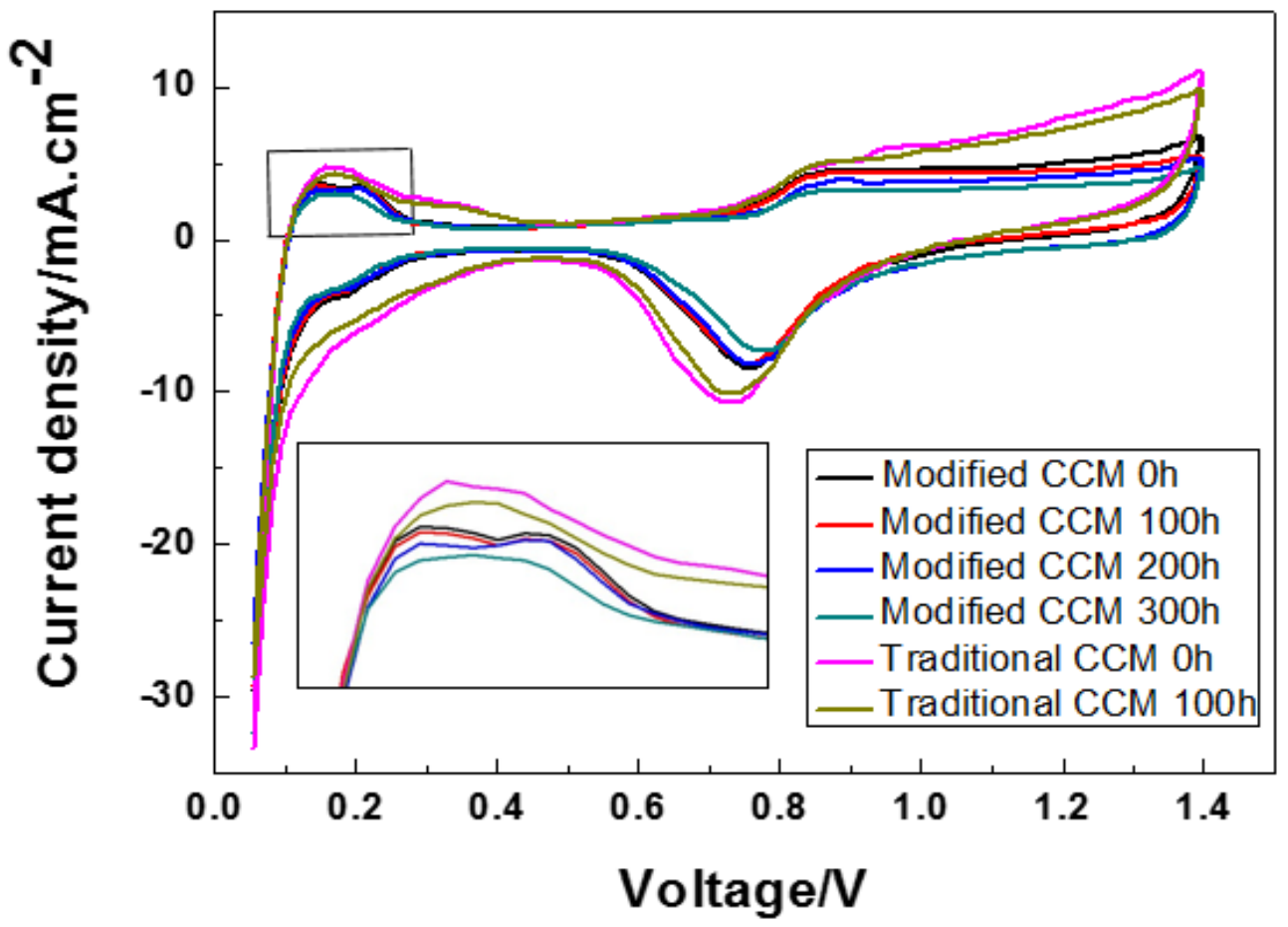

Fig. 5 


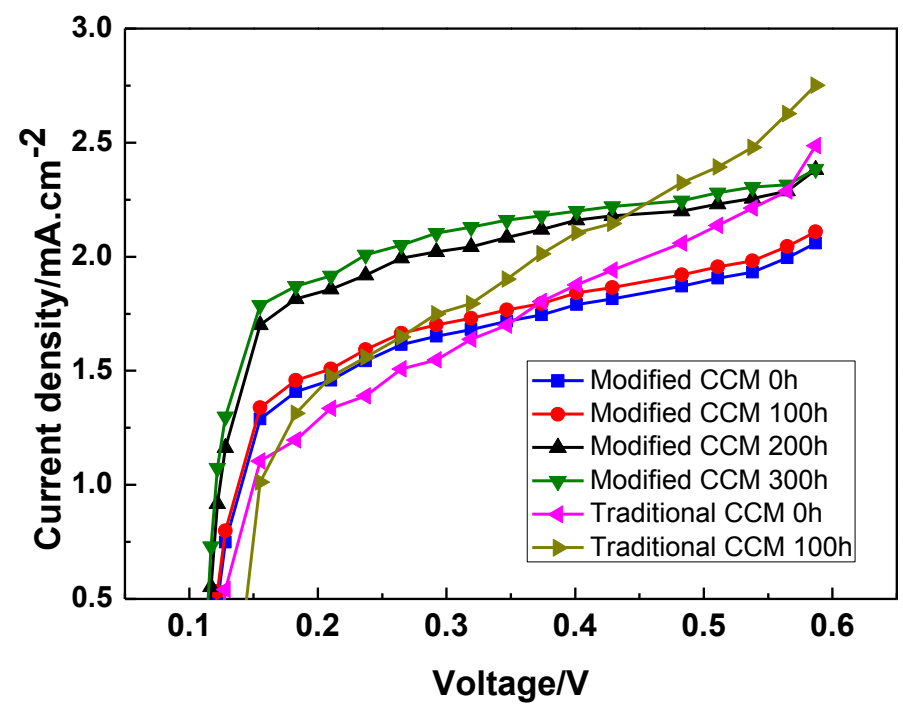

Fig. 6

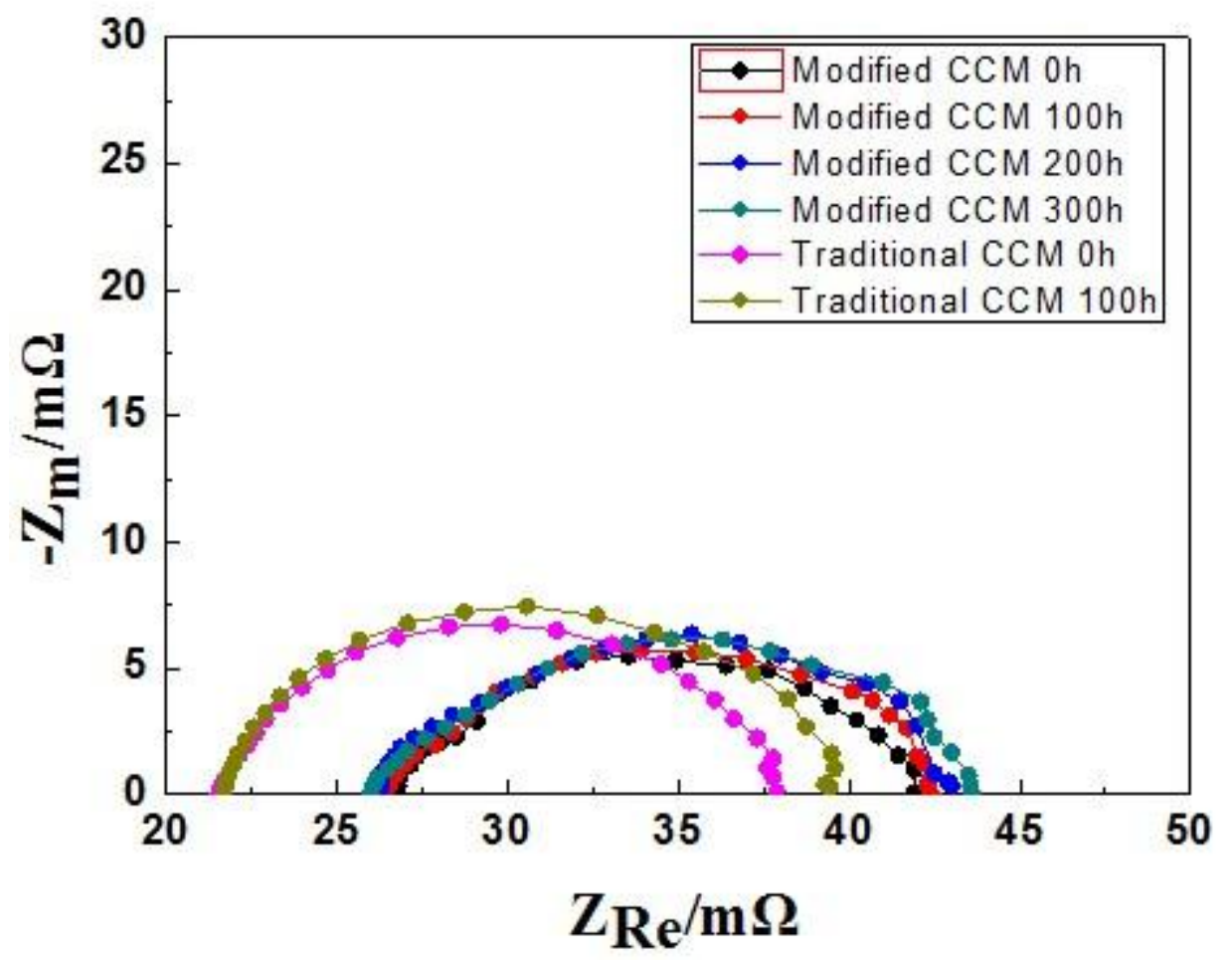

Fig. 7 
Table 1.

\begin{tabular}{|c|c|c|c|c|c|}
\hline $\mathrm{CCM}$ & Membrane & $\begin{array}{c}\text { Cathode Pt loading } \\
\mathrm{mg} / \mathrm{cm}^{2}\end{array}$ & $\begin{array}{c}\text { Anode Pt loading } \\
\mathrm{mg} / \mathrm{cm}^{2}\end{array}$ & Nafion / wt $\%$ & $\begin{array}{c}\text { PTA in catalyst } \\
\text { layer/wt\% }\end{array}$ \\
\hline $\begin{array}{c}\text { Traditional } \\
\text { CCM }\end{array}$ & Nafion XL & 0.4 & 0.2 & 24.5 & 0 \\
\hline $\begin{array}{l}\text { Modified } \\
\text { CCM }\end{array}$ & Nafion XL & 0.4 & 0.2 & 24.5 & 3 \\
\hline
\end{tabular}

Table 2.

\begin{tabular}{|c|c|c|c|c|c|c|c|c|c|}
\hline $\begin{array}{l}\text { Test } \\
\text { point }\end{array}$ & $\begin{array}{l}\text { Current } \\
\text { density } \\
{\left[\mathrm{A} / \mathrm{cm}^{2}\right]}\end{array}$ & $\begin{array}{c}\text { Cell control } \\
\text { temp } \\
{\left[{ }^{\circ} \mathrm{C}\right]}\end{array}$ & $\begin{array}{c}\text { Anode } \mathrm{H}_{2} \\
\text { stoich } \\
{[-]}\end{array}$ & $\begin{array}{c}\text { Anode dew } \\
\text { temp } \\
{ }^{\circ} \mathrm{C}\end{array}$ & $\begin{array}{c}\text { Cathode } \mathrm{O}_{2} \\
\text { stoich } \\
{[-]}\end{array}$ & $\begin{array}{c}{ }_{2} \text { Cathode dew } \\
\text { point } \\
{\left[{ }^{\circ} \mathrm{C}\right]}\end{array}$ & $\begin{array}{l}\text { Inlet } \\
\text { temp } \\
{\left[{ }^{\circ} \mathrm{C}\right]}\end{array}$ & $\begin{array}{c}\text { Test pt. } \\
\text { run time } \\
{[\mathrm{min}]}\end{array}$ & $\begin{array}{c}\text { Outlet } \\
\text { pressure } \\
{[\mathrm{k} \text { Paabs }]}\end{array}$ \\
\hline 1 & 0.02 & 80 & 96 & 68 & 90 & 68 & 85 & 0.5 & 101.3 \\
\hline 2 & 1.2 & 80 & 1.6 & 68 & 1.8 & 68 & 85 & 0.5 & 101.3 \\
\hline 3 & 0.02 & 80 & 96 & 68 & 90 & 68 & 85 & 0.5 & 101.3 \\
\hline 4 & 1.2 & 80 & 1.6 & 68 & 1.8 & 68 & 85 & 0.5 & 101.3 \\
\hline 5 & 0.02 & 80 & 96 & 68 & 90 & 68 & 85 & 0.5 & 101.3 \\
\hline 6 & 1.2 & 80 & 1.6 & 68 & 1.8 & 68 & 85 & 0.5 & 101.3 \\
\hline 7 & 0.02 & 80 & 96 & 68 & 90 & 68 & 85 & 0.5 & 101.3 \\
\hline 8 & 1.2 & 80 & 1.6 & 68 & 1.8 & 68 & 85 & 0.5 & 101.3 \\
\hline 9 & 0.02 & 80 & 96 & 68 & 90 & 68 & 85 & 0.5 & 101.3 \\
\hline 10 & 1.2 & 80 & 1.6 & 68 & 1.8 & 68 & 85 & 0.5 & 101.3 \\
\hline 11 & 0.1 & 80 & 5 & 68 & 5 & 68 & 85 & 0.5 & 101.3 \\
\hline 12 & 0.02 & 80 & 25 & 68 & 25 & 68 & 85 & 0.5 & 101.3 \\
\hline 13 & 0.1 & 80 & 5 & 68 & 5 & 68 & 85 & 0.5 & 101.3 \\
\hline 14 & 0.02 & 80 & 25 & 68 & 25 & 68 & 85 & 0.5 & 101.3 \\
\hline 15 & 0.1 & 80 & 5 & 68 & 5 & 68 & 85 & 0.5 & 101.3 \\
\hline 16 & 0.02 & 80 & 25 & 68 & 25 & 68 & 85 & 0.5 & 101.3 \\
\hline 17 & 0.1 & 80 & 5 & 68 & 5 & 68 & 85 & 0.5 & 101.3 \\
\hline 18 & 0.02 & 80 & 25 & 68 & 25 & 68 & 85 & 0.5 & 101.3 \\
\hline 19 & 0.1 & 80 & 5 & 68 & 5 & 68 & 85 & 0.5 & 101.3 \\
\hline 20 & 0.02 & 80 & 25 & 68 & 25 & 68 & 85 & 0.5 & 101.3 \\
\hline 1 & 0.02 & 80 & 96 & 68 & 90 & 68 & 85 & 0.5 & 101.3 \\
\hline
\end{tabular}


Table 3 .

\begin{tabular}{ccccc}
\hline $\begin{array}{c}\text { EASA } \\
\mathrm{cm}^{2} / \mathrm{mgPt}\end{array}$ & Oh & $100 \mathrm{~h}$ & $200 \mathrm{~h}$ & $300 \mathrm{~h}$ \\
\hline $\begin{array}{c}\text { Traditional } \\
\text { CCM }\end{array}$ & 345.2 & 285.7 & - & - \\
$\begin{array}{c}\text { Modified } \\
\text { CCM }\end{array}$ & 194.4 & 182.5 & 166.7 & 130.9 \\
\hline
\end{tabular}

Table 4.

\begin{tabular}{ccccc}
\hline $\begin{array}{c}\text { charge transfer resistance } \\
\mathrm{m} \Omega\end{array}$ & Oh & $100 \mathrm{~h}$ & $200 \mathrm{~h}$ & $300 \mathrm{~h}$ \\
\hline Traditional CCM & 8.2 & 8.8 & - & - \\
Modified CCM & 7.6 & 7.9 & 8.4 & 8.7 \\
\hline
\end{tabular}

